\title{
Familial aggregation of preeclampsia and intrauterine growth restriction in a genetically isolated population in The Netherlands
}

\author{
Anne L Berends ${ }^{*}{ }^{1}$, Eric A Steegers ${ }^{1}$, Aaron Isaacs ${ }^{2}$, YS Aulchenko ${ }^{2}$, Fan Liu ${ }^{2}$, \\ Christianne J de Groot ${ }^{1,3}$, Ben A Oostra ${ }^{4}$ and Cornelia M van Duijn ${ }^{2}$
}

\begin{abstract}
${ }^{1}$ Department of Obstetrics and Gynaecology, Division of Obstetrics and Prenatal Medicine, University Medical Centre Rotterdam, The Netherlands; ${ }^{2}$ Department of Epidemiology and Biostatistics, University Medical Center Rotterdam, The Netherlands; ${ }^{3}$ Department of Obstetrics and Gynaecology, Haaglanden Medical Centre, The Hague, The Netherlands; ${ }^{4}$ Department of Clinical Genetics, University Medical Centre Rotterdam, The Netherlands
\end{abstract}

Preeclampsia and intrauterine growth restriction are related, pregnancy-specific disorders with a substantial genetic influence, which may have a joint genetic aetiology. We investigated familial aggregation, consanguinity and parent-of-origin effects for preeclampsia and IUGR. Fifty women with previous preeclampsia and 56 with previous pregnancies complicated by intrauterine growth restriction were recruited from a recent genetically isolated population in the Netherlands. Their relationships were estimated by means of a large genealogy database that contains information on more than 110000 individuals from the isolate over $\mathbf{2 3}$ generations. Relationships were quantified using kinship and inbreeding coefficients. Parent-of-origin effects were evaluated by comparing parental kinships. Eighty-six women ( 39 preeclampsia and 47 intrauterine growth restriction) could be linked to one common ancestor within 14 generations. The proportion of related women with previous preeclampsia (95.6\%) or pregnancies complicated by intrauterine growth restriction $(95.1 \%)$ was significantly greater than expected by chance $(P<0.001)$. Combined analysis of both disorders did not change the magnitude of familial aggregation. The proportion of women born from consanguineous marriages was increased in women with previous preeclampsia $(\mathbf{8 1 . 8 \% )}$ ) and those with intrauterine growth restriction $(\mathbf{7 8 \%})$ compared to a random sample $(P<0.001)$. Maternal and paternal kinships were not significantly different in both disorders. We demonstrate cosegregation of preeclampsia and intrauterine growth restriction, supporting a common genetic aetiology. The high proportion of parental consanguineous marriages suggests the possibility of an underlying recessive mutation. No evidence was found for a parent-of-origin effect either in preeclampsia or in intrauterine growth restriction.

European Journal of Human Genetics (2008) 16, 1437-1442; doi:10.1038/ejhg.2008.118; published online 9 July 2008

Keywords: preeclampsia; intrauterine growth restriction; familial aggregation; parent-of-origin effect; genetically isolated population

\footnotetext{
*Correspondence: Dr AL Berends, Department of Obstetrics and Gynaecology, Division of Obstetrics and Prenatal Medicine, Erasmus MC, University Medical Centre Rotterdam, Dr Molewaterplein 60, GJ 3015, Rotterdam, The Netherlands.

Tel: + 31107044 217; Fax: + 31107044 657;

E-mail: a.berends@erasmusmc.nl

Received 25 March 2008; revised 26 May 2008; accepted 29 May 2008; published online 9 July 2008
}

\section{Introduction}

Preeclampsia is a pregnancy-specific disease and the leading cause of maternal and fetal morbidity and mortality worldwide. ${ }^{1}$ It is defined by de novo hypertension and proteinuria. Although maternal symptoms present in 
the second and third trimester of pregnancy, preeclampsia finds its origin in early placentation. Its exact aetiology remains unknown, but shallow endovascular trophoblast invasion and inadequate uteroplacental artery remodelling resulting in malperfusion of the placenta are key pathologic features of preeclampsia. ${ }^{2}$ Similar placental pathology was previously associated with (idiopathic) intrauterine growth restriction (IUGR), ${ }^{2,3}$ a condition affecting fetal growth without appreciable maternal disease. The shared placental pathology has led to the hypothesis that preeclampsia and IUGR are related conditions, yet with different clinical manifestations. ${ }^{4}$

A familial component, long recognized in the aetiology of preeclampsia, ${ }^{5}$ supports the hypothesis of a genetic origin. Different modes of inheritance were proposed over the years varying from single gene models ${ }^{5}$ to complex segregation involving both maternal and fetal genotypes. ${ }^{5}$ Additionally, there is substantial evidence for familial clustering $^{6,7}$ of IUGR, with both maternal and paternal genes contributing to the IUGR phenotype. ${ }^{6}$ In addition to Mendelian modes of inheritance, alternative genetic mechanisms such as parent-of-origin effects were proposed for both preeclampsia and IUGR. ${ }^{8,9}$ Imprinted genes play a fundamental role in the regulation of human growth and disruption of these genes may cause growth disturbances as is seen in uniparental disomies. ${ }^{9}$ Recently, dysregulation of imprinted genes was reported in human placental tissue of IUGR pregnancies. ${ }^{10}$ Evidence supporting the role of imprinting in preeclampsia was described in mutant mice models. ${ }^{11}$ In humans, the STOX1 gene was identified as a putative imprinted gene for preeclampsia following matrilineal inheritance. ${ }^{12}$ Thus far, however, neither the role of STOX1 in preeclampsia nor the evidence for imprinting could be confirmed in other populations. ${ }^{13-15}$

Whereas familial aggregation is well established for both preeclampsia and IUGR, a question that remains to be answered is whether a joint genetic aetiology underlies the common pathology of both disorders. Further, the evidence for parent-of-origin effects in preeclampsia is still scarce and not replicated. Availability of genealogical information over multiple generations is helpful to assess familial clustering of these disorders for which absolute recurrence risks are low. We investigated familial aggregation of preeclampsia and IUGR in an isolated population for which extensive genealogical data were available. In addition, we studied parent-of-origin effects, which may indicate genomic imprinting and inbreeding effects, suggestive of recessive forms of disease.

\section{Methods \\ Population}

The study was conducted in a genetically isolated population in the southwest of the Netherlands, ${ }^{16}$ and is part of a larger research programme called Genetic Research in
Isolated Populations (GRIP), which aims to identify genetic factors in the development of complex disorders. ${ }^{17}$ This population was founded around 1750 and has been characterized by minimal inward migration until the period 1960-1970 and rapid population growth over the last two centuries. Currently, the population consists of $>20000$ individuals. ${ }^{16}$ The Erasmus Medical Ethical Committee of the Erasmus Medical Centre Rotterdam approved the study protocol.

\section{Participants}

Women with a history of preeclampsia or IUGR pregnancy were selected from the GRIP area. Ascertainment of participants has been described in detail elsewhere. ${ }^{13}$ Briefly, 50 women with pregnancies complicated by preeclampsia and 56 with IUGR pregnancies were included in the study. Women were identified from National Birth Registration Records dating from 1983 up to 2004. Diagnoses were confirmed by the research physician after reviewing the medical records. Only women who were living in the isolate at the time of delivery were included in the study.

Preeclampsia was defined as de novo hypertension (systolic $\geq 140$ and/or diastolic $\geq 90 \mathrm{~mm} \mathrm{Hg}$ ) and proteinuria $\geq 300 \mathrm{mg}$ per $24 \mathrm{~h}$, or at least $1+$ on semiquantitative analysis after 20 weeks gestation. Superimposed preeclampsia was defined as new-onset proteinuria after 20 weeks of gestation in women with chronic hypertension. Preeclampsia was defined as 'early onset' when it was diagnosed before 34 weeks of gestation, and as 'late onset' when diagnosed after 34 weeks. IUGR was defined as a newborn birth weight equal to or below the fifth percentile for gestational age at delivery, according to the Dutch fetal growth charts. ${ }^{18}$ If preeclampsia and IUGR cooccurred, women were categorized in the preeclampsia group. Women who gave birth to children with congenital anomalies were excluded from the study group. Only singleton pregnancies were included. Women were invited to participate in the study by their general practitioner or obstetrician. All participants provided written informed consent.

\section{Genealogy}

Genealogical data, comprised of the names, dates, and places of birth and death of relatives were obtained from the participants by questionnaires. Municipal and church registers and data from a large genealogy database containing information on more than 110000 individuals from the GRIP region were used to extend this pedigree information up to 23 generations.

The relationship between two individuals can be expressed as the pairwise kinship coefficient. This is the probability that a randomly drawn allele from one person is identical by descent with a randomly drawn allele at the same locus of another person. The probability that two 
alleles in one individual are identical by descent is expressed as the inbreeding coefficient. This coefficient represents the degree of consanguinity between the parents of this individual.

Pairwise kinship and inbreeding coefficients were calculated for a subset of women with previous preeclampsia or IUGR pregnancies. Those with both parents born outside the isolate were excluded from analyses as genealogical information is available only for people born in the isolate.

\section{Parent-of-origin effects}

We evaluated parent-of-origin effects in a subset of women who could be linked to a single common ancestor. We tested whether women were more often related through the paternal or maternal lineage by comparing kinship coefficients for the maternal line to that of the paternal line. ${ }^{19}$ Under the null hypothesis of no parent-of-origin effect, the average degree of relationship of the fathers should not differ from that of the mothers.

\section{Statistical analysis}

General characteristics were compared between groups using Student's t-test for continuous variables and $\chi^{2}$ statistics for dichotomous variables with SPSS 11.0.1 for Windows. We calculated pairwise kinship and inbreeding coefficients using PEDIG software. ${ }^{20}$ This method is based on the same rationale such as standard 'heritability' estimate methods. We studied whether kinship (K) and inbreeding (I) coefficients in women with previous preeclampsia or IUGR pregnancies deviated from the expected by chance. We made three categories for $\mathrm{K}: \mathrm{K}$ between $1 / 2$ and $1 / 2^{6}$ denoting those related within three generation; $K<1 / 2^{6}$ denoting those related more distantly than three generations; and $K=0$ not related. The frequencies of pairs in each category were calculated. Similarly, we categorized the inbreeding coefficients, I between $1 / 2$ and $1 / 2^{6}, I<1 / 2^{6}$ and $I=0$, denoting close, remote, and no inbreeding, respectively. Women with previous preeclampsia and with previous IUGR pregnancies were analysed as separate groups as well as one combined group. To test for evidence for familial aggregation, we performed Monte Carlo analysis. For each patient group, randomly chosen age and sex-matched control groups were drawn from the genealogical database of this genetically isolated population. Thousand replicas were used to estimate a null distribution of kinship and inbreeding coefficients reflecting the baseline level in this population. Empirical $P$-values were estimated from these distributions. The same procedure was performed to generate the null distribution of the parental kinships.

\section{Results \\ Genealogy}

A total of 106 women were included in the study. Fifty had previous preeclampsia, and 56 had previous IUGR pregnancies. After extensive genealogical analysis, 39 of 50 (78\%) women with a history of preeclampsia could be linked to a common ancestor over 14 generations. For IUGR, 47 of 56 (84\%) women could be similarly linked. After pooling the two groups of patients, 86 (81\%) could be linked to a single ancestor, which was a significantly greater number than was expected by chance $(56 \%, P$-value $<0.001)$. Supplementary Figure 1 depicts the pedigree linking these 86 women. General descriptions for the total groups are presented in Table 1 . There were no significant clinical differences between those connected and those not connected to a common ancestor.

\section{Familial aggregation}

Seventy-four women (33 with previous preeclampsia and 41 with previous IUGR pregnancies) met the criterion of having at least one parent born in the isolate. We tested whether these women were more closely related than expected by chance, adjusting for age and sex (Table 2). The proportion of women that was related $(K>0)$ in the preeclampsia group (95.6\%) was higher than expected (62.7\%; $P<0.001)$. In the IUGR group, this proportion was

Table 1 Description of women in the total study group and of those who could be and could not be linked to a common ancestor

\begin{tabular}{|c|c|c|c|c|c|c|}
\hline Characteristics & $\begin{array}{l}P E \text { total } \\
N=50\end{array}$ & $\begin{array}{l}\text { PE with common } \\
\text { ancestor } \\
N=39\end{array}$ & $\begin{array}{l}P E \text {, no common } \\
\text { ancestor } \\
N=11\end{array}$ & $\begin{array}{c}\text { IUGR total } \\
N=56\end{array}$ & $\begin{array}{c}\text { IUGR with common } \\
\text { ancestor } \\
N=47\end{array}$ & $\begin{array}{c}\text { IUGR, no common } \\
\text { ancestor } \\
N=9\end{array}$ \\
\hline $\begin{array}{l}\text { Birth weight of newborns (g) } \\
\text { Early preeclampsia } \\
\text { Preeclampsia and IUGR } \\
\text { co-occurence }\end{array}$ & $\begin{array}{c}2559(886) \\
16(32) \\
8(16)\end{array}$ & $\begin{array}{r}2594(876) \\
11(28.2) \\
6(15.4)\end{array}$ & $\begin{array}{r}2435(954) \\
5(45.5) \\
2(18.2)\end{array}$ & $\begin{array}{c}2223(547) \\
\text { NA } \\
\text { NA }\end{array}$ & $\begin{array}{c}2240(544) \\
N A \\
N A\end{array}$ & $\begin{array}{c}2136(587) \\
N A \\
N A\end{array}$ \\
\hline
\end{tabular}

IUGR, intrauterine growth restriction; NA, not applicable; $\mathrm{PE}$, preeclampsia.

Values are presented as means (SD) or absolute numbers (\%). 
Table 2 Distribution of kinship coefficients for women with previous PE or IUGR pregnancies and controls

\begin{tabular}{|c|c|c|c|c|c|c|}
\hline Kinship coefficient & $P E$ & Controls & $I U G R$ & Controls & $P E+I U G R$ & Controls \\
\hline $\begin{array}{l}1 / 2-1 / 2^{6} \\
<1 / 2^{6} \\
0 \\
\text { Total number of pairs }\end{array}$ & $\begin{array}{l}0.9(5) \\
94.7(500)^{\mathrm{a}} \\
4.4(23)^{\mathrm{a}} \\
100(528)\end{array}$ & $\begin{array}{r}0.6 \\
62.1 \\
37.3 \\
100\end{array}$ & $\begin{array}{l}1.0(8) \\
94.1(772)^{\mathrm{a}} \\
4.9(40)^{\mathrm{a}} \\
100(820)\end{array}$ & $\begin{array}{r}0.6 \\
56.6 \\
42.8 \\
100\end{array}$ & $\begin{array}{l}1.1(29) \\
94.2(2544)^{\mathrm{a}} \\
4.7(128)^{\mathrm{a}} \\
100(2701)\end{array}$ & $\begin{array}{r}0.6 \\
58.8 \\
40.6 \\
100\end{array}$ \\
\hline
\end{tabular}

IUGR, intrauterine growth restriction; PE, preeclampsia.

All values are percentages with absolute number between parentheses.

${ }^{\text {a } P}<0.001$ compared with controls.

Table 3 Distribution of kinship and inbreeding coefficients for women with previous IUGR pregnancies who did not smoke during pregnancy

\begin{tabular}{lcc}
\hline Coefficient & $\begin{array}{c}\text { Kinship } \\
\text { coefficients }\end{array}$ & $\begin{array}{c}\text { Inbreeding } \\
\text { coefficients }^{\mathrm{b}}\end{array}$ \\
\hline $1 / 2-1 / 2^{6}$ & $0.5(1)$ & $0(0)$ \\
$<1 / 2^{6}$ & $96.2(202)$ & $76.2(16)$ \\
0 & $3.3(7)$ & $23.8(5)$ \\
Total number of & $100(210)$ & $100(21)$ \\
pairs/absolute number & & \\
\hline
\end{tabular}

IUGR, intrauterine growth restriction.

All values are percentages with absolute number between parentheses. ${ }^{a}$ Kinship coefficients were not significantly different from the complete group IUGR.

Inbreeding coefficients were not significantly different from the complete group IUGR.

95.1\%, which was also significantly increased (57.2\%; $P<0.001)$. Given the possible confounding effect of smoking on birth weight, we analysed $\mathrm{K}$ also after exclusion of those who reported smoking during pregnancy. The proportion of related women did not significantly change $(96.7 \% ; P=0.5$, Table 3$)$. When preeclampsia and IUGR were analysed as a combined group, $95.3 \%$ were related as compared to the expected $59.4 \%(P<0.001)$. In addition, significantly more women with previous preeclampsia and IUGR pregnancies could be linked to one common ancestor (97\%) than the expected by chance $(66 \%, P<0.001)$. We further compared kinship coefficients between women with early and late-onset preeclampsia. No significant differences were found.

\section{Consanguinity and parent-of -origin effects}

Next, we calculated the proportion of women born from consanguineous marriages $(I>0)$ (Table 4$)$. For preeclampsia, this proportion was $81.8 \%$, which was significantly higher than expected by chance $(38.8 \% ; P<0.001)$. In the IUGR group (78 versus $35.4 \%$ ) and combined groups (79.7 versus $36.5 \%)$, these proportions were also significantly increased $(P<0.001)$. Additional analyses in those women of the IUGR group who did not smoke during pregnancy showed similar proportions $(76.2 \% ; P=0.6$, Table 3$)$. To evaluate the evidence of parent-of-origin effects, we compared the mean kinship of the mothers and fathers of related women. For women with previous preeclampsia, the ratio of mean maternal kinship to paternal kinship was 0.91 . This ratio was not significantly different from the ratio expected by chance $(P=0.50)$, implying no evidence of parent-of-origin effect. For IUGR, we found a ratio of 1.01 , which was not significantly different either $(P=0.30)$. Finally, we evaluated consanguinity and parent-of-origin effects in subsets of women with early and late-onset preeclampsia. In these groups, the ratio of mean maternal kinship and paternal kinship was not significantly different from controls.

\section{Discussion}

As expected, we found significant evidence for familial aggregation for women with previous preeclampsia and IUGR pregnancies. The analysis of preeclampsia and IUGR separately or pooled yielded no significant differences in magnitude of familial aggregation. Pooling two disorders with different genetic origins should result in a smaller proportion of related pairs if the two traits are independent. As the proportion of related pairs in the pooled group was similar to that of the separate patient groups, our data suggest that preeclampsia and IUGR cosegregate in families and probably have a common genetic origin. This finding corroborates the hypothesis of a, at least to some extend, joint aetiology of preeclampsia and IUGR. ${ }^{4}$ To our knowledge, no other studies have investigated familial aggregation of preeclampsia and IUGR in a similar way. It was, however, previously reported that women being born small for gestational age have an increased risk to develop preeclampsia, ${ }^{21}$ which is also compatible with a joint genetic origin. Already in 1977, a common pathophysio$\operatorname{logy}^{2}$ was suggested as morphologic examinations of placental bed biopsies indicated similar pathological changes in preeclampsia and IUGR. ${ }^{3}$ Additional evidence for this hypothesis comes from the observation that both disorders share common risk factors ${ }^{4}$ and, as has become evident over the last years, are both associated with similar long-term disease such as an increased risk of cardiovascular disease. ${ }^{22,23}$ In this cohort, we have reported earlier that more than $40 \%$, and almost $30 \%$ of the women with previous pregnancies complicated by preeclampsia or IUGR, respectively were diagnosed with chronic 
Table 4 Distribution of inbreeding coefficients for women with previous PE or IUGR pregnancies and controls

\begin{tabular}{lcccccc}
\hline Inbreeding coefficient & $P E$ & Controls & IUGR & Controls & PE+IUGR & Controls \\
\hline Close inbreeding & $3.0(1)$ & 3.2 & $2.4(1)$ & 3.2 & $2.7(2)$ & 3.2 \\
Remote inbreeding & $78.8(26)^{\mathrm{a}}$ & 35.6 & $75.6(31)^{\mathrm{a}}$ & 32.2 & $77.0(57)^{\mathrm{a}}$ & 33.3 \\
No inbreeding & $18.2(6)^{\mathrm{a}}$ & 61.2 & $22.0(9)^{\mathrm{a}}$ & 64.6 & $20.3(15)^{\mathrm{a}}$ & 63.5 \\
Total number & $100(33)$ & 100 & $100(41)$ & 100 & $100(74)$ & 100 \\
\hline
\end{tabular}

IUGR, intrauterine growth restriction; NA, not applicable; PE, preeclampsia.

All values are percentages with absolute number between parentheses.

${ }^{\mathrm{a}} P<0.001$ compared with controls.

hypertension. ${ }^{24}$ This association could not be explained by admixture.

An increased number of women with previous preeclampsia and IUGR pregnancies were born from consanguineous marriages. This proportion suggests a recessive mode of inheritance, at least in a subset of these women. This is in agreement with several previous studies suggesting recessive inheritance for preeclampsia. ${ }^{5}$ Given the high risk of chronic hypertension later in life, ${ }^{24}$ this finding is in line with the higher prevalence of consanguinity in parents of patients with chronic hypertension. ${ }^{25}$ However, two earlier studies that compared the incidence of consanguinity in women with preeclampsia and non-hypertensive pregnancies, could not confirm any impact of consanguinity on the occurrence of preeclampsia. ${ }^{26,27}$ An important difference between these studies and ours is that consanguinity was based on self-reports instead of on extensive genealogical data, which did not allow for analyses of distant relationships. Regarding IUGR, one large study proposed a recessive inheritance pattern in a subset of their families, yet the majority of the families would best fit a dominant model. ${ }^{7}$ A recent large scale study conducted in Lebanon indicated that consanguinity is associated with decreased birth weight. ${ }^{28}$ Alternatively, the increased number of women born from consanguineous marriages is also consistent with a polygenic mode of inheritance, particularly in an inbred population.

We also used our genealogic database to evaluate the evidence for parent-of-origin effects in the transmission of preeclampsia or IUGR in a subset of women that could be linked to a common ancestor. No evidence for a parent-oforigin effect was found either for women with a history of preeclampsia or in those with IUGR pregnancies. These findings are in accordance with our previous findings that no maternal preferential transmission of STOX1 gene in women with preeclampsia and IUGR was observed. ${ }^{13}$ However, our observations are at odds with prior findings of a maternal parent-of-origin effect in Dutch preeclampsia patients. ${ }^{8}$ Differences between the study of Oudejans et $a l^{8}$ and our study may be explained by the selection of patients. Oudejans et $a l^{8}$ included affected sib pairs of whom a majority were born to mothers who experienced preeclampsia- or pregnancy-induced hypertension. ${ }^{8}$ In this way, they aimed to target a familial form of preeclampsia with early onset disease. This highly selected group represents only a small proportion of the overall group of preeclamptic women. As we included an unbiased series of women with preeclampsia, our study may have lacked statistical power to pick up the effect of such a small subgroup. The analysis of a subgroup of our patients with early and late-onset preeclampsia similarly yielded no evidence for a parent-of-origin effect. Moreover, we found no differences in the degree of relationship between early and late-onset preeclampsia, although it was previously suggested that familial aggregation is stronger for the clinically more severe type of preeclampsia that often manifests early in pregnancy. ${ }^{29}$

The strength of our study is the availability of an extensive genealogical database. Most of the previous studies on familial aggregation and parent-of-origin effects are based on two or three generations..$^{5,7,8,29}$ The availability of the genealogy revealed familial aggregation in seemingly unrelated cases. The extended pedigree containing a large proportion of the women with previous preeclampsia and IUGR may prove to be helpful in future in discovering genes involved in the pathogenesis of both disorders.

Our study also has limitations. The IUGR phenotype was defined as small for gestational age babies. We based our definition on birth weight, because ultrasound examination was not widely used at the time of delivery of the women that we studied. This may have resulted in the inclusion of newborns that were constitutionally small, but not growth restricted. However, by using the stringent criterion of birth weight equal or below the fifth percentile, we aimed to minimize misclassification. Familial aggregation of IUGR owing to environmental factors such as severe nutritional deficiencies or smoking cannot be excluded with certainty, but the impact of malnutrition on birth weight in studies within the developed countries is not likely to be large. Regarding smoking, reanalysis of kinship and inbreeding coefficients after exclusion of women who reported smoking during pregnancy did not change our results.

Finally, studying patients in an isolated population may raise the question whether the findings can be generalized to the population at large. However, as our population is of more recent isolation, the genetic makeup may more closely resemble that of the general population. $^{30}$ Further, our simulation studies based on the 
genealogy have shown that this potential problem concerns primarily rare variants. ${ }^{30}$ For common genetic variants, our simulation studies show that no substantial differences between the GRIP isolate and the general population are expected. ${ }^{30}$ This is in line with the finding that common variants identified in isolates such as Iceland and Sardinia are found with similar frequencies and effect in outbred populations.

In summary, we found evidence for familial aggregation in women with previous preeclampsia and IUGR pregnancies in a genetically isolated population. The increased frequency of parental consanguineous marriages suggests that the recessive mutations play a role. Further, we observed cosegregation of preeclampsia and IUGR, supporting the hypothesis of a common genetic pathogenesis. No evidence was found for a parent-of-origin effect either in preeclampsia or in IUGR.

\section{Acknowledgements}

The authors would like to thank the participants of the study. Petra Veraart is acknowledged for her contribution in the genealogy. Wilma Keller, Pieter Snijders, Richard Pal and Roger Heijdanus are acknowledged for their help in the ascertainment of the participants. This study was supported by an Erasmus Medical Centre Grant and by the Centre for Medical Systems Biology (CMSB).

\section{Competing interests}

None.

\section{References}

1 Ness RB, Roberts JM: Heterogeneous causes constituting the single syndrome of preeclampsia: a hypothesis and its implications. Am J Obstet Gynecol 1996; 175: 1365-1370.

2 Kaufmann P, Black S, Huppertz B: Endovascular trophoblast invasion: implications for the pathogenesis of intrauterine growth retardation and preeclampsia. Biol Reprod 2003; 69: $1-7$.

3 Brosens I, Dixon HG, Robertson WB: Fetal growth retardation and the arteries of the placental bed. Br J Obstet Gynaecol 1977; 84: $656-663$.

4 Ness RB, Sibai BM: Shared and disparate components of the pathophysiologies of fetal growth restriction and preeclampsia. Am J Obstet Gynecol 2006; 195: 40-49.

5 Lachmeijer AM, Dekker GA, Pals G, Aarnoudse JG, ten Kate LP, Arngrimsson R: Searching for preeclampsia genes: the current position. Eur J Obstet Gynecol Reprod Biol 2002; 105: 94-113.

6 Svensson AC, Pawitan Y, Cnattingius S, Reilly M, Lichtenstein P: Familial aggregation of small-for-gestational-age births: the importance of fetal genetic effects. Am J Obstet Gynecol 2006; 194: $475-479$.

7 Ghezzi F, Tibiletti MG, Raio L et al: Idiopathic fetal intrauterine growth restriction: a possible inheritance pattern. Prenat Diagn 2003; 23: 259-264.

8 Oudejans CB, Mulders J, Lachmeijer AM et al: The parentof-origin effect of 10q22 in pre-eclamptic females coincides with two regions clustered for genes with down-regulated expression in androgenetic placentas. Mol Hum Reprod 2004; 10: 589-598.
9 Devriendt K: Genetic control of intra-uterine growth. Eur J Obstet Gynecol Reprod Biol 2000; 92: 29-34.

10 McMinn J, Wei M, Schupf $\mathrm{N}$ et al: Unbalanced placental expression of imprinted genes in human intrauterine growth restriction. Placenta 2006; 27: 540-549.

11 Kanayama N, Takahashi K, Matsuura $\mathrm{T}$ et al: Deficiency in p57Kip2 expression induces preeclampsia-like symptoms in mice. Mol Hum Reprod 2002; 8: 1129-1135.

12 van Dijk M, Mulders J, Poutsma A et al: Maternal segregation of the Dutch preeclampsia locus at 10q22 with a new member of the winged helix gene family. Nat Genet 2005; 37: 514-519.

13 Berends AL, Bertoli-Avella AM, de Groot CJ, van Duijn CM, Oostra BA, Steegers EA: STOX1 gene in pre-eclampsia and intrauterine growth restriction. BJOG 2007; 114: 1163-1167.

14 Iglesias-Platas I, Monk D, Jebbink J et al: STOX1 is not imprinted and is not likely to be involved in preeclampsia. Nat Genet 2007; 39: 279-280; author reply 280-281.

15 Kivinen K, Peterson H, Hiltunen L et al: Evaluation of STOX1 as a preeclampsia candidate gene in a population-wide sample. Eur J Hum Genet 2007; 15: 494-497.

16 Aulchenko YS, Heutink P, Mackay I et al: Linkage disequilibrium in young genetically isolated Dutch population. Eur J Hum Genet 2004; $12: 527-534$

17 Sayed-Tabatabaei FA, van Rijn MJ, Schut AF et al: Heritability of the function and structure of the arterial wall: findings of the Erasmus Rucphen Family (ERF) study. Stroke 2005; 36: 2351-2356.

18 Kloosterman GJ: On intrauterine growth. The significance of prenatal care. Int J Gynaecol Obstet 1970; 8: 895-912.

19 Hoppenbrouwers IA, Liu F, Aulchenko YS et al: Maternal transmission of multiple sclerosis in a dutch population. Arch Neurol 2008; 65: 345-348.

20 Boichard D: PEDIG: A FORTRAN package for pedigree analysis studied for large populations. In Proceeding of the Seventh World Congress Genet. Appl Livest Prod CD-ROM Communication 2002; No. 28-13, 2002.

21 Innes KE, Marshall JA, Byers TE, Calonge N: A woman's own birth weight and gestational age predict her later risk of developing preeclampsia, a precursor of chronic disease. Epidemiology 1999; 10: $153-160$.

22 Bellamy L, Casas JP, Hingorani AD, Williams DJ: Pre-eclampsia and risk of cardiovascular disease and cancer in later life: systematic review and meta-analysis. BMJ 2007; 335: 974.

23 Smith GD, Harding S, Rosato M: Relation between infants' birth weight and mothers' mortality: prospective observational study. BMJ 2000; 320: 839-840.

24 Berends AL, de Groot CJ, Sijbrands EJ et al: Shared constitutional risks for maternal vascular-related pregnancy complications and future cardiovascular disease. Hypertension 2008; 51: 1034-1041.

25 Rudan I, Smolej-Narancic N, Campbell H et al: Inbreeding and the genetic complexity of human hypertension. Genetics 2003; 163: $1011-1021$.

26 Badria LF, Abu-Heija A, Zayed F, Ziadeh SM, Alchalabi H: Has consanguinity any impact on occurrence of pre-eclampsia and eclampsia? J Obstet Gynaecol 2001; 21: 358-360.

27 Stevenson AC, Davison BC, Say B et al: Contribution of fetal/ maternal incompatibility to aetiology of pre-eclamptic toxaemia. Lancet 1971; 2: 1286-1289.

28 Mumtaz G, Tamim H, Kanaan M et al: Effect of consanguinity on birth weight for gestational age in a developing country. Am J Epidemiol 2007; 165: 742-752.

29 Skjaerven R, Vatten LJ, Wilcox AJ, Ronning T, Irgens LM, Lie RT: Recurrence of pre-eclampsia across generations: exploring fetal and maternal genetic components in a population based cohort. BMI 2005; 331: 877.

30 Pardo LM, MacKay I, Oostra B, van Duijn CM, Aulchenko YS: The effect of genetic drift in a young genetically isolated population. Ann Hum Genet 2005; 69: 288-295. 